\title{
ON CHARACTERISTIC PROPERTIES OF HARMONIC FUNCTIONS ${ }^{1}$
}

\author{
E. F. BECKENBACH
}

1. Introduction. Green's Theorem for functions of two variables,

$$
\begin{aligned}
\iint_{R}\left(\frac{\partial u}{\partial x} \frac{\partial v}{\partial x}+\frac{\partial u}{\partial y} \frac{\partial v}{\partial y}\right) d x d y+\iint_{R} v\left(\frac{\partial^{2} u}{\partial x^{2}}+\frac{\partial^{2} u}{\partial y^{2}}\right) d x d y \\
=\int_{C} v \frac{\partial u}{\partial n} d s,
\end{aligned}
$$

yields several characteristic integral properties of harmonic functions, that is, of functions $u(x, y)$ for which

$$
\frac{\partial^{2} u}{\partial x^{2}}+\frac{\partial^{2} u}{\partial y^{2}}=0 .
$$

From (1) we obtain, for harmonic functions $u(x, y)$,

$$
\int_{c} \frac{\partial u}{\partial n} d s=0
$$

whence the mean value properties,

$$
u\left(x_{0}, y_{0}\right)=\frac{1}{2 \pi} \int_{0}^{2 \pi} u\left(x_{0}+r \cos \theta, y_{0}+r \sin \theta\right) d \theta,
$$

(4) $u\left(x_{0}, y_{0}\right)=\frac{1}{\pi r^{2}} \int_{0}^{2 \pi} \int_{0}^{r} u\left(x_{0}+\rho \cos \theta, y_{0}+\rho \sin \theta\right) \rho d \rho d \theta$,

follow easily. Equation (1) yields also

$$
\int_{C}\left(u \frac{\partial v}{\partial n}-v \frac{\partial u}{\partial n}\right) d s=0
$$

for pairs of harmonic functions. Further, if $u(x, y)$ is harmonic, then for each $v(x, y)$ of class $C^{\prime}$ in $R$, and of class $C^{0}$ in $R+C$, and satisfying $v(x, y)=0$ on $C$, we have, from (1),

$$
\iint_{R}\left(\frac{\partial u}{\partial x} \frac{\partial v}{\partial x}+\frac{\partial u}{\partial y} \frac{\partial v}{\partial y}\right) d x d y=0
$$

Presented to the Society, November 26, 1949; received by the editors February 4, 1952.

1 This research was supported in part by the Office of Naval Research. 
Finally, (1) yields

$$
\iint_{R}\left[\left(\frac{\partial u}{\partial x}\right)^{2}+\left(\frac{\partial u}{\partial y}\right)^{2}\right] d x d y=\int_{C} u \frac{\partial u}{\partial n} d s
$$

for harmonic functions $u(x, y)$.

Harmonic functions can be characterized in terms of each of the above properties. Bôcher [4] and Koebe [7] independently established the result that (2) characterizes harmonic functions. For (3) and (4), Levi [8] and Tonelli [10] proved the converse of the Gauss mean value theorem; further, the equality of the two mean value functions in (3) and (4) characterizes harmonic functions in the class of continuous functions $[2 ; 3]$, and characterizes almost harmonic functions in the class of summable functions [1]. Gergen [5] showed that (5) characterizes harmonic functions $u(x, y)$ in terms of any given harmonic function $v(x, y)$ for which

$$
v(x, y) \neq 0 \text {. }
$$

And it is well known in relation to the Dirichlet principle (see, for instance [6, p. 417]), that if $u(x, y)$ is of class $C^{\prime}$ in $R+C$, and satisfies (6) for each $v(x, y)$ of class $C^{\prime}$ in $R+C$, with

$$
v(x, y)=0
$$

on $C$, then $u(x, y)$ is harmonic in $R$; this follows trivially from (1) if $u(x, y)$ is given to be class $C^{\prime \prime}$, whence the more general result can be established, for instance, by means of averaging functions.

We shall show that, under either of two additional assumptions, the first of which corresponds to Gergen's condition (8), property (7) also characterizes harmonic functions.

2. Theorem. Letting $o\left(r^{\alpha}\right)$ denote a function (not always the same function) $\phi(r)$ such that

$$
\lim _{r \rightarrow 0} \frac{\phi(r)}{r^{\alpha}}=0
$$

we shall establish the following result.

Theorem 1. If $u(x, y)$ is of class $C^{\prime}$ in a domain $D$, and for each $\left(x_{0}, y_{0}\right)$ in $D$ we have

$$
\int_{0}^{2 \pi} \int_{0}^{r}\left[\left(\frac{\partial u}{\partial x}\right)^{2}+\left(\frac{\partial u}{\partial y}\right)^{2}\right] \rho d \rho d \theta-\int_{0}^{2 \pi} u \frac{\partial u}{\partial n} r d \theta=o\left(r^{2}\right),
$$

where the integrals are taken over the area and circumference, respectively, 
of the circle with center at $\left(x_{0}, y_{0}\right)$ and radius $r$, and if

$$
u(x, y) \neq 0
$$

in $D$, then $u(x, y)$ is harmonic in $D$.

Proof. By hypothesis, for each point $\left(x_{0}, y_{0}\right)$ of $D$ we have

$$
u(x, y)=u\left(x_{0}, y_{0}\right)+a r \cos \theta+b r \sin \theta+o(r),
$$

where $a$ and $b$ denote $\partial u / \partial x$ and $\partial u / \partial y$, respectively, evaluated at $\left(x_{0}, y_{0}\right)$. Hence

$$
\begin{aligned}
& \frac{\partial u}{\partial x}=a+o(1), \quad \frac{\partial u}{\partial y}=b+o(1), \\
& \frac{\partial u}{\partial n}=a \cos \theta+b \sin \theta+o(1)
\end{aligned}
$$

and

$u \frac{\partial u}{\partial n}=u\left(x_{0}, y_{0}\right) \frac{\partial u}{\partial n}+a^{2} r \cos ^{2} \theta+2 a b r \cos \theta \sin \theta+b^{2} r \sin ^{2} \theta+o(r)$,

so that

$$
\int_{0}^{2 \pi} \int_{0}^{r}\left[\left(\frac{\partial u}{\partial x}\right)^{2}+\left(\frac{\partial u}{\partial y}\right)^{2}\right] \rho d \rho d \theta=\pi r^{2}\left(a^{2}+b^{2}\right)+o\left(r^{2}\right),
$$

and

$$
\int_{0}^{2 \pi} u \frac{\partial u}{\partial n} r d \theta=u\left(x_{0}, y_{0}\right) \int_{0}^{2 \pi} \frac{\partial u}{\partial n} r d \theta+\pi r^{2}\left(a^{2}+b^{2}\right)+o\left(r^{2}\right) .
$$

It follows from (10), (12), and (13) that

$$
u\left(x_{0}, y_{0}\right) \int_{0}^{2 x} \frac{\partial u}{\partial n} r d \theta=o\left(r^{2}\right) .
$$

By Saks' generalization [9] of the above-mentioned theorem of Bôcher and Koebe, if $u(x, y)$ is of class $C^{\prime}$ in a domain, and

$$
\int_{0}^{2 \pi} \frac{\partial u}{\partial n} r d \theta=o\left(r^{2}\right)
$$

for each point of the domain, then $u(x, y)$ is harmonic there. Accordingly, the theorem follows from (11) and (14).

3. Lemma. We shall use the following result. 
Lemma. If $u(x, y)$ is of class $C^{\prime}$ in a domain $D$, and for each $\left(x_{0}, y_{0}\right)$ in $D$ either

(i) there is a neighborhood of $\left(x_{0}, y_{0}\right)$ in which $u(x, y)$ is harmonic, or

(ii) $u\left(x_{0}, y_{0}\right)=0$,

and if $u(x, y)$ has summable second-order partial derivatives $\partial^{2} u / \partial x^{2}$ and $\partial^{2} u / \partial y^{2}$ in $D$, then $u(x, y)$ is harmonic in $D$.

In the lemma the assumption concerning second derivatives perhaps is redundant; but the function $u(x, y) \equiv|x|$ shows that this is not true of the assumption that $u(x, y)$ is of class $C^{\prime}$.

Proof of Lemma. We shall prove the lemma by establishing (6) for an arbitrary circular disc $R$, with circumference $C$, in $D$; this proof was kindly supplied by Professor L. V. Ahlfors.

Let the set of points of $R+C$ for which (ii) is satisfied be denoted by $F$, and let the complement of $F$ in $R+C$ be denoted by $E$. For any horizontal line-segment $t$ in $R+C$ let the intersections of $t$ with $E$ and $F$ be denoted by $E_{t}$ and $F_{t}$, respectively. Then $F_{t}$ is closed and therefore consists of a denumerable set $S_{t}$ and the set $L_{t}$ of the limit points of $F_{t}$; either of the sets $E_{t}$ and $F_{t}$ might of course be null.

Since (ii) holds at each point of the horizontal set $F_{t}$, it follows that $\partial u / \partial x=0$ on $L_{t}$. Further, since by hypothesis $\partial^{2} u / \partial x^{2}$ is summable in $R+C$, it is summable on almost all horizontal line-segments $t$ in $R+C$. Hence, integrating by parts and using the fact that (9) holds on $C$, we have

$$
\int_{t} \frac{\partial u}{\partial x} \frac{\partial v}{\partial x} d x=-\int_{E_{t}} v \frac{\partial^{2} u}{\partial x^{2}} d x
$$

whence

$$
\iint_{R} \frac{\partial u}{\partial x} \frac{\partial v}{\partial x} d A=-\int\left[\int_{E t} v \frac{\partial^{2} u}{\partial x^{2}} d x\right] d y=-\iint_{E} v \frac{\partial^{2} u}{\partial x^{2}} d A,
$$

by Fubini's theorem. Similarly,

$$
\iint_{R} \frac{\partial u}{\partial y} \frac{\partial v}{\partial y} d A=-\iint_{E} v \frac{\partial^{2} u}{\partial y^{2}} d A .
$$

Adding (15) and (16), we obtain (6) from the fact that (i) holds at each point of $E$.

4. Theorem. If the assumption concerning second derivatives is redundant in the lemma, then it is redundant also in the following result; in that case, condition (11) could simply be omitted in the statement of Theorem 1. 
Theorem 2. If $u(x, y)$ is of class $C^{\prime}$ in a domain $D$, and (10) holds for each $\left(x_{0}, y_{0}\right)$ in $D$, and if $u(x, y)$ has summable second-order partial derivatives $\partial^{2} u / \partial x^{2}$ and $\partial^{2} u / \partial y^{2}$ in $D$, then $u(x, y)$ is harmonic in $D$.

Proof. By Theorem $1, u(x, y)$ is harmonic in the part of $D$ where $u(x, y) \neq 0$; then Theorem 2 follows from the lemma.

\section{REFERENCES}

1. E. F. Beckenbach, On almost subharmonic functions, Revista, Serie A, Universidad Nacional de Tucuman vol. 4 (1944) pp. 243-254.

2. E. F. Beckenbach and T. Rad6, Subharmonic functions and surfaces of negative curvature, Trans. Amer. Math. Soc. vol. 35 (1933) pp. 662-674.

3. E. F. Beckenbach and Maxwell Reade, Mean-values and harmonic polynomials, Trans. Amer. Math. Soc. vol. 53 (1943) pp. 230-238.

4. M. Bôcher, On harmonic functions in two dimensions, Proceedings of the American Academy of Arts and Sciences vol. 41 (1906) pp. 577-583.

5. J. J. Gergen, Note of a theorem of Bôcher and Koebe, Bull. Amer. Math. Soc. vol. 37 (1931) pp. 591-596.

6. A. Hurwitz and R. Courant, Funktionentheorie, Berlin, Springer, 1925.

7. P. Koebe, Herleitung der partiellen Differentialgleichung der Potentialfunktion aus Integraleigenschaft, Sitzungsberichte der Berliner Mathematischen Gesellschaft vol. 5 (1906) pp. 39-42.

8. E. Levi, Sopra una proprietd caratteristica delle funzioni armoniche, Atti della Reale Accademia Lincei vol. 18 (1909) pp. 10-15.

9. S. Saks, Note on defining properties of harmonic functions, Bull. Amer. Math. Soc. vol. 38 (1932) pp. 380-382.

10. L. Tonelli, Sopra una proprietd caratteristica delle funzioni armoniche, Atti della Reale Accademia Lincei vol. 18 (1909) pp. 577-582.

University of California, Los Angeles, and INSTITUTE FOR ADVANCEd STUDY 